\title{
Stimulus probability and same-different classification
}

\author{
ROBERT G. PACHELLA and JEFFREY 0. MILLER \\ University of Michigan, Ann Arbor, Michigan 48105
}

\begin{abstract}
Three experiments investigated the effect of stimulus probability on same-different classification time. In Experiments I and II, subjects made same responses on the basis of name matches of simultaneously presented letters. Half of the same trials involved letters that were also physically identical. Experiment I showed that the presentation probability of specific letters affected name matches and different respenses, but not physical matches. Experiment II varied stimulus contrast as well as probability. Contrast had a main effect but did not interact with probability at any level of processing. In Experiment III, subjects were switched to the physical level of processing. Stimuli that now had the same name but differed in case were called different. In this condition, the probability effects observed in Experiment II disappeared. These results are interpreted as demonstrating that stimulus probability has its effect during the process that derives the name of the stimulus from the visual representation. This process takes place before the name comparison is made, and the name comparison process precedes the determination of the different response.
\end{abstract}

Many theoretical models have appeared recently that describe the information processing in simple experimental tasks as a series of temporally discrete mental operations or processing stages. A number of these models have proposed that the end result of the encoding stages of processing is the establishment of an internal code, which is then utilized in all further cognitive activity. For example, Theios (1973) has hypothesized that the result of the encoding process is stimulus identification. This encoded representation is then used in the response determination stages of processing, which, depending upon the particular experimental task, can consist of various cognitive activities such as scanning a memory buffer to see if the stimulus character is a positive or a negative item or in activating the overt response that names the stimulus.

Wattenbarger (1970, see also Nickerson, 1971, for a discussion of Wattenbarger's work) has also presented a model which clearly illustrates this kind of encoding process. He presented data suggesting that the processing involved in memory scanning consists of an encoding stage which converts the visual stimulus into an internal analogue of the stimulus's name. This is followed by a memory scanning stage which compares the name of the stimulus to the names of the items held in memory.

A key feature of processing schemes of this type is the notion that encoding involves a transformation of

This research was supported by the Advanced Research Projects Agency, U.S. Department of Defense, and monitored by the U.S. Air Force Office of Scientific Research, under Contract No. F44620-72-C-0019 with the University of Michigan, Human Performance Center. Experiments $I$ and II were originally presented at the Twelfth Annual Meeting of the Psychonomic Society. St. Louis, Missouri, November 1973. Requests for reprints should be sent to Robert G. Pachella, Human Performance Center. 330 Packard Road. Ann Arbor. Michigan 48104. the form of the stimulus representation. Encoding converts the stimulus representation from a form that has properties like the proximal or retinal stimulus into a form that is consistent with some permanently stored internal representation. This internal representation is often thought of as a cognitive label with properties analogous to the name of the stimulus, as in Wattenbarger's model, but it could just as well be some internal code like a memory address or some kind of internal template with properties analogous to an external stimulus.

One important consequence of this notion that encoding transforms the stimulus representation from an external to an internal code is that many factors whose effects are localized at a point prior to the transformation should have effects which are independent of factors whose effects are localized after the transformation. For example, specific conditions of stimulus presentation, like stimulus clarity, should be independent of specific conditions of response determination, like the number of items in a memory buffer, since in converting to a stored representation the effects of the conditions of stimulus presentation have been removed from the internal representation. Thus, if the encoding processes result in a representation that is analogous to the name of the stimulus, then the effects of conditions of stimulus presentation, like clarity, should be independent of any subsequently effective factors.

The Theirs (1973) model is a case in point. This model localizes the effect of stimulus probability on reaction time in the response determination or memory scanning stages of processing. These stages follow the encoding processes, which have resulted in an identification of the stimulus. Therefore, the model should predict that stimulus probability and stimulus clarity should have additive effects on reaction time. 
Miller and Pachella (1973) recently collected data that are relevant to this particular issue. They varied stimulus probability and stimulus clarity in a memory scanning task and found these factors to interact. This result indicates that wherever stimulus probability has its effect, it must be at a point where the effect can be modified by the level of stimulus degradation. In other words, either stimulus probability has part of its effect prior to identification of the stimulus or identification does not take place before memory scanning. While either case is inconsistent with the Theios model, on the basis of Wattenbarger's data and other data presented by Klatzky and Smith (1972), Miller and Pachella opted for the former possibility-that at least some of the effect of stimulus probability is localized during the encoding stages of processing.

The purpose of the present experiments was to examine further the nature of a possible effect of stimulus probability on stimulus encoding. Subjects were presented with letter pairs, whose probability of occurrence was varied. Their task was to classify these letters into same or different pairs on the basis of name identity (Experiments I and II) or physical identity (Experiment III). This classification task was chosen for two reasons. First, it minimizes the necessity of memory comparison processes (as in the memory scanning paradigm) upon which stimulus probability may also have large and systematic effects (Theios, Smith, Haviland, Traupmann, \& Moy, 1973). Secondly, by way of physical level vs. name level comparisons as in the studies of Posner and Mitchell (1967), the simultaneous comparison task allows for a somewhat finer dissection of stimulus encoding, for the better localization of the effects of experimental variables.

\section{EXPERIMENT I}

\section{Method}

A PDP-1 computer controlled the presentation of stimuli and recorded the responses and response latencies. The stimuli were letter pairs presented on a CRT, with each member of the pair enclosed in a rectangle. The letters were vector generated and were white against a black background. The rectangles were about $10 \mathrm{~cm}$ high and $6 \mathrm{~cm}$ wide, and about $5 \mathrm{~cm}$ apart. Within each rectangle was a letter, either uppercase or lowercase. Uppercase letters were about $7.5 \mathrm{~cm}$ high, while lowercase letters were about $3.75 \mathrm{~cm}$ high. The particular letters used in this experiment were A, G, M, and E. They were chosen because their upper- and lowercase forms were easily discriminable from each other. Subjects sat about $65 \mathrm{~cm}$ from the CRT, and responses were made with the right and left index fingers, which were placed on a microswitch keyboard directly in front of the subject.

Each of 28 right-handed subjects was run in 10 blocks of 44 trials. Stimulus probability was held constant over all 10 blocks. For half of the subjects. the high-probability letters were $A$ and $G$ and the low-probability letters were $M$ and $E$, while for the other half of the subjects these probabilities were reversed. On each trial, the stimulus consisted of two physically identical letters, two letters differing only in case, or two different letters of the same probability (either high or low). There were no trials on which a high-probability letter was paired with a low-probability letter. High-probability letters occurred on approximately $82 \%$ of the trials, with low probability letters occurring on the remaining $18 \%$. Half of the trials were same trials, half were different. Also, there were equal numbers of physical identity matches and name identity matches. Within each condition, all of the possible permutations of upper-vs. lowercase and left vs. right member of letter pair were counterbalanced. The subjects for whom $A$ and $G$ were the frequent letters made the same response with their right hands; the others made the same response with their left hands.

Subjects were instructed that they would see letter pairs, and that they should make the "same" response if the two letters had the same name, and the different response otherwise. Subjects were told to respond as fast as they could. being careful to make very few errors. At the beginning of each block, the subjects were given a ready signal. Stimuli remained on the screen until a response was made, which was then followed by accuracy feedback. About $550 \mathrm{msec}$ after the response, the next stimulus appeared.

\section{Results}

For each block, the average RT and percent error were computed at each level of probability, for each type of trial (i.e., physical match, name match, different). These values were averaged across subjects and blocks, and the averages are plotted in Figure 1. An analysis of variance was done on RTs. Both the main effects of probability $[\mathrm{F}(1,26)=6.2, \mathrm{p}<.025]$ and type of trial $[F(2.52)=91.52, p<.001]$ were significant. The only significant interaction was between group (whether AG or ME were the frequent letters) and probability, apparently because one of these pairs was much easier than the other. An analysis of variance of the error rates showed the main effects of practice $[F(9,234)=5.9, p<.001]$ and type

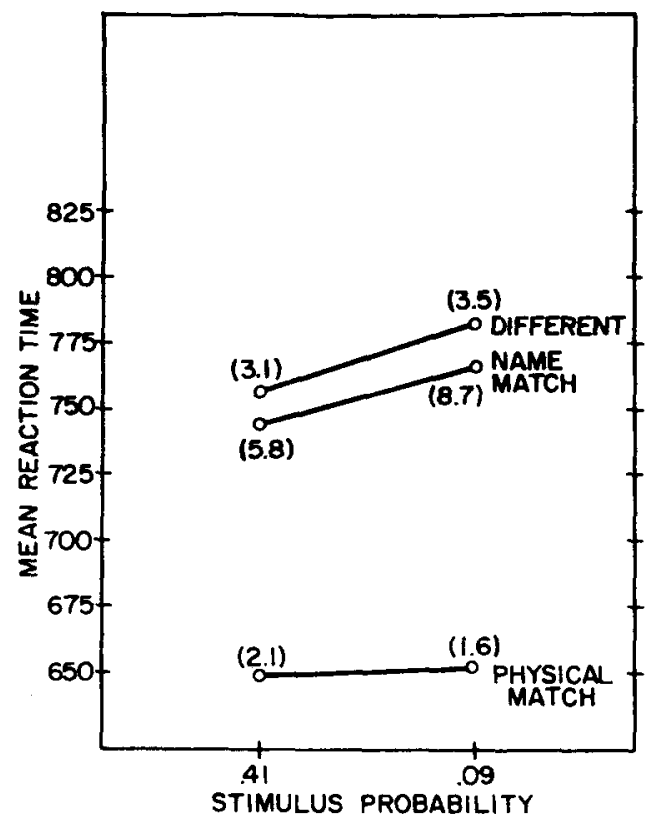

Figure 1. Reaction time as a function of stimulus probability and trial type for Experiment $I$. Numbers in parentheses indicate the perceptage of error for each condition. 
of trial $[F(2,24)=26.8, p<.001]$ to be significant. The main effect of probability did not quite attain significance $[F(1,26)=3.04, p<.10]$, but the interaction between probability and type of trial did $[F(2,52)=3.63, p<.05]$. This was the only significant interaction.

Thus, stimulus probability has an effect on the comparison of simultaneously presented letter pairs. As shown in Figure 1, the effect seems to be localized entirely on the name match and different responses, since the difference in reaction time as a function of probability for physical matches is extremely small (649 msec vs. $653 \mathrm{msec})$. The weight of this conclusion needs to be attenuated somewhat since the interaction of type of trial and probability on reaction time does not quite attain significance, but the error data indicate the difference to be real.

\section{EXPERIMENT II}

The purpose of the second experiment was to replicate the first experiment and to include a condition with a degraded stimulus presentation. The question of interest here was to see if the probability effect noted in Experiment I would be modified by the level of stimulus contrast, as occurred in the memory scanning and naming experiments of Miller and Pachella (1973).

\section{Method}

The procedure used in Experiment II was very similar to that used in Experiment I, with the following exceptions. Sixteen unpracticed subjects were run in 14 blocks of 44 trials. The blocks alternated between high-and low-contrast stimuli. The high-contrast stimuli were essentially identical to those in Experiment I. The low-contrast stimuli were produced by simply turning down the contrast on the CRT. The amount of attenuation was approximately $1.5 \mathrm{log}$ units, and the same low level of contrast was maintained on all low-contrast blocks for all subjects. The stimulus letters used in this experiment were $D, G, H$, and $M$, and the subjects were randomly assigned to one of four groups so that the combincations DG and HM, and DH and GM, could be used, with each pair being the high-probability pair for one of the groups. All subjects were right-handed and all made the "same" response with their right hands.

\section{Results}

For each block, the average RT and percent error were computed at each level of stimulus probability for each type of trial. These values are averaged across subjects and blocks within each level of stimulus contrast and are plotted in Figure 2. An analysis of variance on the $R T$ s showed that the main effects of contrast $[F 1,12)=28.05, \mathrm{p}<.001]$, group $[\mathrm{F}(3,12)$ $=3.71, \mathrm{p}<.05]$, practice $[\mathrm{F}(6,72)=3.35$, $\mathrm{p}<.005]$, probability $[\mathrm{F}(1,12)=7.68, \mathrm{p}<.025]$, and type of trial $[F(2,24)=61.56, p<.001]$ were significant. The significant interactions were those between group and type of trial $[F(6,24)=3.84$, $\mathrm{p}<.01]$, probability and type of trial $[\mathrm{F}(2,24)=$ $10.61, \mathrm{p}<.005]$, and the three-way interaction of groups, type of trial, and probability $[F(6,24)=5.37$,

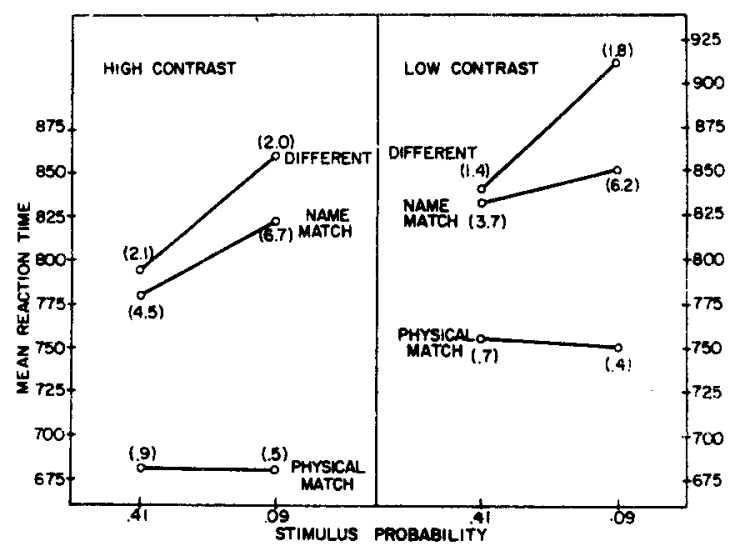

Figure 2. Reaction time as a function of stimulus probability and trial type for two levels of stimulus contrast in Experiment $I$. Numbers in parentheses indicate the percentage of error for each condition.

$p<.01]$. Analysis of variance on the errors showed that the significant main effects were practice $[F(6,76)$ $=4.62, \mathrm{p}<.01]$ and type of trial $[\mathrm{F}(2,24)=12.5$, $\mathrm{p}<.001]$. The only significant interaction in the error data was that between group, type of trial, and probability $[F(6,24)=3.73, \mathrm{p}<.01]$.

The data in the left panel of Figure 2 show that Experiment I was nicely replicated. In fact, the right panel, i.e., the low-contrast condition, also replicates the basic finding of Experiment $I$. This is substantiated by the finding that the factor of contrast has only a significant main effect in the overall analysis of variance and does not interact with any other variable. Furthermore, when these two, essentially identical, replications are collapsed over the contrast variable, the two-way interaction between trial type and probability becomes significant. Thus, the tentative conclusion reached in Experiment I, that probability affects only name matches and different trials, is greatly strengthened.

The second purpose of Experiment II was to look at the nature of the relationship between stimulus probability and contrast. This inquiry can be facilitated by replotting the data from Figure 2 so that the degradation by probability interaction can be viewed separately for each trial type. This is done in Figure 3.

The effects of probability and degradation are essentially additive at each processing level. This conclusion is supported by the following statistics: As noted above, neither the three-way interaction of contrast, probability and trial type nor the overall two-way interaction of contrast and probability is significant $[F(2,24)=.46$ and $F(1,12)=.46$, respectively]. Additionally, none of the simple two-way interactions of contrast and probability at each level of trial type are significant [for the physical match, $F(1,15)=.02$; for the name match, $F(1,15)=$ 1.27; and for the different trials, $F(1,15)=1.01$ ]. 


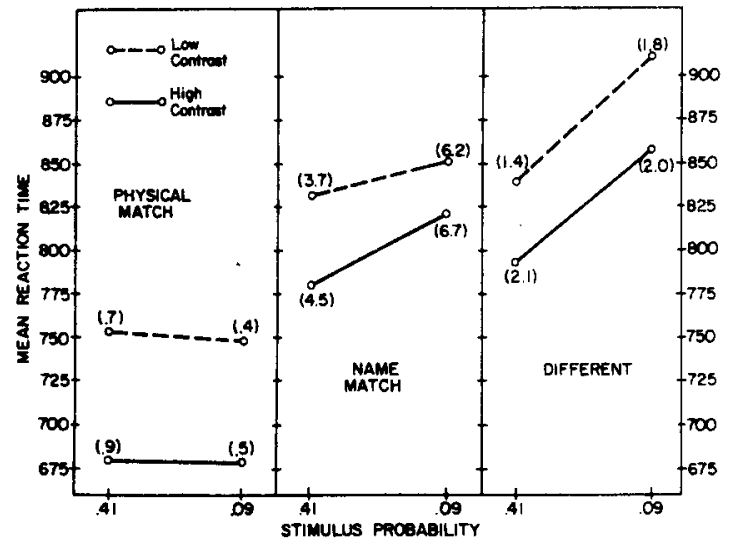

Figure 3. Reaction time as a function of stimulus probability and stimulus contrast for each trial type in Experiment II. Numbers in parentheses indicate the percentage of error for each condition.

Further, the root mean square deviations (RMSD) from the best fitting additive models for each trial type are extremely small. For the physical match and the different trials, the RMSDs are less than $1 \mathrm{msec}$, and for the name match trials, the RMSD is just $6 \mathrm{msec}$. Thus, the additivity of the probability and contrast effects at each level of processing can be fairly safely assumed.

One last feature of these data, which will be of some importance in later discussion, should be noted. The overall two-way interaction between contrast and type of trial failed to reach significance $[\mathrm{F}(2,24)=2.88$, $p>.05]$. However, the case for the additivity of these two factors cannot be made as strongly here as it was above, since there is a sizable (although statistically insignificant) difference in the contrast effect for each trial type. The size of the contrast effect for physical matches is $72 \mathrm{msec}$, while for name matches and different trials it is 40 and $49 \mathrm{msec}$, respectively.

\section{EXPERIMENT III}

The purpose of Experiment III was to see if the effect of probability observed in Experiments I and II was simply due to the lack of physical identity on name match and different trials or rather to the necessity of having to make same responses to letters that in fact were not physically identical (i.e., name matches).

\section{Method}

All of the procedural details of Experiment III were identical to those of Experiment II with the exception that 24 unpracticed subjects were run instead of 16 . The important difference in this experiment, however, was instructional. Subjects were instructed to use physical identity as the criterion for a same response. Letter pairs which now had the same name, but differed in case (e.g., Dd), were to be classitied as different. Since exactly the same input sequences were presented to the subjects, this resulted in an imbalance in the response probabilities. In the present experiment, $25 \%$ of the responses (i.e., the physical matches) were same and $75 \%$ were different (same-name, different-case trials and different-name trials). In the present context, it was felt that it was better to keep the proportions of each type of stimulus trial identical to those of Experiment II rather than response probability.

\section{Results}

For each block, the mean RT and percent error were computed at each level of stimulus probability for each type of trial. These values were averaged across subjects and blocks within the levels of stimulus contrast, and the results are shown in Figure 4. The analysis of variance for the RT data revealed only one significant main effect. That was for the effect of degradation $[F(1,20)=87.87, p<.01]$. The main effect of probability was not significant $[F(1,20)=2.30, \mathrm{p}>.10]$. The only significant interaction was that of Practice by Degradation $[F(6,120)=5.06, p<.01]$, practice having a larger effect on degraded stimuli than on clear stimuli. With regard to error rate, analysis of variance showed only significant effects of degradation $[F(1,20)=7.90$, $\mathrm{p}<.01]$ and trial type $[\mathrm{F}(2,40)=16.66, \mathrm{p}<.01]$.

The conclusion can, thus, be asserted, on the basis of these data, that switching subjects to the physical level of judging sameness greatly reduces the previously observed effect of probability on both the same-name and different-trial types. Likewise, this manipulation removes the reaction time differences between trial types. Here too, however, we might attenuate this conclusion somewhat, since trial type differences are still apparent in the error rates and the particular manipulation used involved unbalancing response probability.

\section{GENERAL DISCUSSION}

The data of the three experiments presented in this paper are completely consistent with the notion

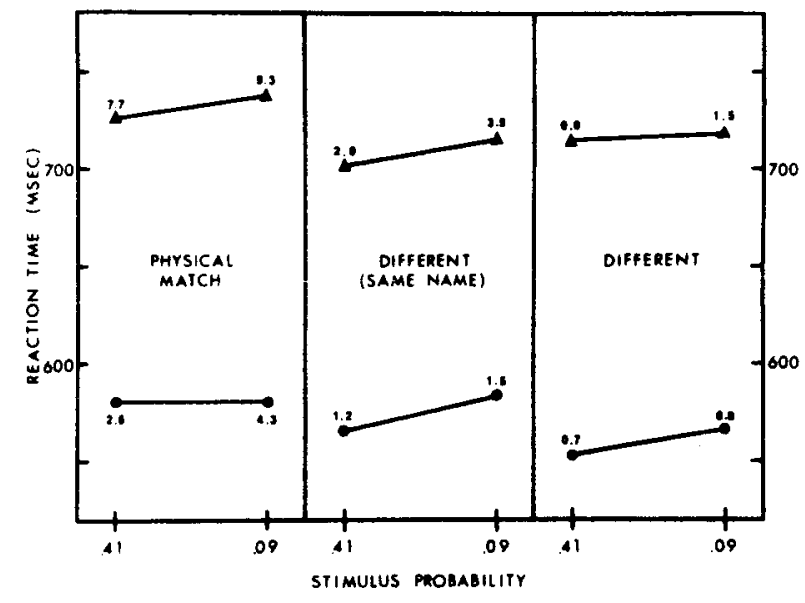

Figure 4. Reaction time as a function of stimulus probability and stimulus contrast (circles represent high contrast and triangles low contrast) for each trial type in Experiment III. Numbers above each point indicate percentage of error for each condition. 
suggested by Miller and Pachella (1973) that there exists a locus of stimulus probability effects within the stimulus encoding stages of processing. The more precise locus of these effects seems to be the stages that necessitate the conversion of physical representations into the name of the stimulus. Conversion stages such as these have also characterized the encoding process in the models of memory scanning and choice RT developed by Wattenbarger (1970) and Theios (1973).

There exist some inconsistencies between these notions and other. previously published ideas that need further discussion. however. First, the data are not entirely consistent with those of Miller and Pachella (1973). The present investigators had anticipated on the basis of their earlier work that stimulus probability would affect the name level of processing. However, it was also anticipated on the basis of the earlier work that the effect of probability would be magnified by degrading the stimulus presentation. In other words, the conclusion of Miller and Pachella (1973) that stimulus probability affected the encoding stage was based upon the interaction of probability and degradation. No such interactions have been found anywhere in the present experiments.

One argument that can be constructed to account for the lack of interaction in the present task would be to conceive that in the letter matching task the effects of degradation are removed from the stimulus representation by some stage prior to the process that obtains the names of the letters. One candidate for such an early process might be the physical comparison process itself. If the physical comparison process preceded the name comparison process, then the effects of degradation would likely be removed as a result of testing for the physical identity of the stimulus. An important prediction from this conception is the fact that the size of the degradation effect should then be constant across all trial types. This follows from the fact that once the reaction time difference due to the degradation of the stimulus appears at the physical comparison level. it must be passed along to all subsequent stages. More generally, this relation would hold for any model of processing that supposed an early (i.e.. prior to any comparison processes) stage that removed the effects of stimulus degradation.

The present data are consistent with such notions. although they could be much stronger. Consistent with the hypothesis is the fact. as noted earlier. that the Contrast by Trial Type interaction in Experiment II did not reach significance. However, this null hypothesis. that the contrast differences across trial types are equal, should be accepted only with the greatest trepidation in light of the differences that can be observed in the data (see Figure 3). It should be further noted in this regard that the observed insignificant difference is one of "underadditivity." that is. the faster trial type (physical comparison) shows a larger contrast effect than the slower trials (name comparisons). Posner (1973, p. 99) has argued that a significant difference of this type ${ }^{1}$ is indicative of the temporal independence of the physical and name levels of processing. The precision of the present data, at best, leaves this still an open question.

A similar issue concerns the temporal independence of the sameness vs. the difference levels of processing. Here. the present data create a more persuasive argument. Several authors (Bamber, 1969; Beller, 1970; Krueger, 1970) have proposed that the process of testing for sameness and the process of testing for differemce are not serially dependent upon each other: They have suggested that the tu'o processes proceed from stimulus onset independently of one another and that the response is contingent upon which process terminates first. In the context of the present data, the alternative argument seems more compelling; that difference testing follows sameness processing and is contingent upon it. If probability effects are introduced at the point in time where the name of the stimulus is acquired (i.e., during the name comparison process), and if this process precedes the determination of the different response, then one would expect to find the same magnitude of probability effect at this point in time and also for the subsequent different-response determination. More importantly, a manipulation that removed the necessity for acquiring the name of the stimulus, should remove the probability effect, not only from the immediately affected stage, but also from all subsequent stages. This is one of the strongest predictions that can be made on the basis of what has been classically called "subtractive" logic (see Pachella. 1974). The present experiments for the most part conform to this pattern. Probability effects appear in both Experiments I and II at the name level and the different level of processing. In Experiment I, they are also of the same magnitude at both levels; in Experiment II, particularly for the low-contrast condition. the additivity is more questionable. Most importantly, however, both effects disappear when the subjects perform a task that does not require the acquisition of the name of the stimulus. Thus, it would appear that stimulus probability affects the name level of comparison, and that the effects that are produced there are passed on to the subsequent stage that determines the different response. If the name acquisition stage is eliminated. then the probability effects previously noted on the different trials disappear.

Finally, the relation of the present data to some recently published data involving stimulus probability in letter classification tasks should be briefly noted. Nickerson (1973) observed stimulus probability effects on physically identical stimulus pairs in a sequential letter matching task involving only uppercase letters. Since case was not manipulated. subjects could have 
used the efficient strategy of basing their judgments solely on the physical identity of the stimuli. However. since case was not manipulated, it is impossible to know whether subjects in fact employed this strategy. Alternatively, the subjects could have based their judgments on the names of the stimuli. It seems reasonable that, given no instructions to the contrary, the sequential presentation of the letters may have led the subjects to code the letters by name. The present experiments indicate that such a strategy would lead to probability effects for letters that were physically identical.

Similarly, Krueger (1973) used a task very similar to that of the present Experiment III and observed no stimulus probability effects on same trials, but obtained probability effects on different trials. Again, however, no manipulation of letter case was used in this study, so it is impossible to know whether or not different responses were based on the simple lack of physical identity or on the fact that the stimuli had different names. In the latter case, Krueger's experiment becomes similar to Experiment II of the present paper, in which probability effects on different responses of the same magnitude as Krueger's were obtained. The occurrence of trials in the present Experiment III that have the same name but must be responded to as different (e.g., Aa) forces the subject to base his response on the simple lack of physical identity in which case the probability effects on different responses disappear. Without the manipulation of case in letter classification studies such as these, it is impossible to determine objectively the psychological strategy employed by the subjects, and as the present experiments demonstrate, it is this strategy that determines the nature of stimulus probability effects.

\section{REFERENCES}

BAMBER, D. Reaction times and error rates for "same"-"different" judgments of multidimensional stimuli. Perception \& Psychophysics, 1969, 6, 169-174.
Beller, H. K. Parallel and serial stages in matching. Journal of Experimental Psychology, 1970, 84, 213-219.

KLAtzky. R. L., \& Smith, E. E. Stimulus expectancy and retrieval from short-term memory. Journal of Experimental Psychology, 1972, 94, 101-107.

KRUEGER, L. E. Effect of bracketing lines on speed of "same""different" judgment of two adjacent letters. Journal of Experimental Psychology, 1970, 84, 324-330.

KRUEGER. L. E. Effect of stimulus frequency on speed of "same""different" judgments. In S. Kornblum (Ed.), Attention and performance IV. New York: Academic Press, 1973.

Miller, J. O., \& Pachella, R. G. Locus of the stimulus probability effect. Journal of Experimental Psychology, 1973. 101, 227.231

Nickerson, R. Binary classification reaction time: A review of some studies of human information processing capabilities. Psychonomic Monograph Supplements, 1971, 4(Whole No. 65).

NiCKERSON. R. Frequency, recency and repetition effects on same and different response times. Journal of Experimental Psychology, 1973, 101, 330-336.

Pachella, R. G. The interpretation of reaction time in information processing research. In B. Kantowitz (Ed.), Human information processing: Tutorials in performance and cognition. Hillsdale, N.J: Lawrence Erlbaum Associates, 1974.

Posner, M. I. Cognition: An introduction. Glenview, Ill: Scott, Foresman, 1973.

Posner, M. I., \& Mitchell, R. F. Chronometric analysis of classification. Psychological Review, 1967, 74, 392-409.

Theios, J. Reaction time measurements in the study of memory processes: Theory and data. In G. Bower (Ed.), The psychology of learning and motivation: Advances in research and theory (Vol. 7). New York: Academic Press, 1973.

Theios, J., Smith, P., Haviland, S., Traupmann, J., \& MoY, M. Memory scanning as a serial, self-terminating process. Journal of Experimental Psychology, 1973, 97, 323-336.

WATTENBARGER, B. L. The representation of the stimulus in character classification. Unpublished doctoral dissertation. University of Michigan, 1970.

\section{NOTE}

1. Posner cites an unpublished masters thesis by $S$. Bugge that in conception sounds similar to the present Experiment II. In that study, the stimulus "degradation" consisted of letter rotation, as opposed to contrast. and a significant underadditivity of Rotation by Trial Type resulted.

(Received for publication June 16, 1975; revision received September 8, 1975.) 